\title{
AUTOPRESENTACIÓN PERFECCIONISTA DURANTE LA INFANCIA Y SU ASOCIACIÓN CON ANSIEDAD ESCOLAR
}

\author{
María del Pilar Aparicio-Flores \\ Departamento de Psicología Evolutiva y Didáctica, Facultad de Educación, \\ Universidad de Alicante \\ pilar.aparicio@ua.es \\ María José Hernández-Amorós \\ Departamento de Didáctica General y Didácticas Específicas, Facultad de Educación, \\ Universidad de Alicante \\ María Encarnación Urrea-Solano \\ Departamento de Didáctica General y Didácticas Específicas, Facultad de Educación, \\ Universidad de Alicante \\ Aitana Fernández-Sogorb \\ Departamento de Psicología Evolutiva y Didáctica, Facultad de Educación, \\ Universidad de Alicante
}

\section{RESUMEN}

La Autopresentación Perfeccionista (APP), conocida como el deseo de proyectar una imagen perfecta hacia los demás con características protectoras de su exhibición social y con la necesidad reiterativa de divulgarse excelente, es uno de los factores más influyentes en cuanto a psicopatologías derivadas y menos estudiado en el contexto educativo. El objetivo de este estudio consistió en examinar la relación entre la APP y la Ansiedad Escolar en una muestra compuesta por 860 participantes con edades comprendidas entre 8 y 12 años $(M=9.86$; $D E=1.26)$, matriculados en centros públicos y privados de la provincia de Alicante. Se empleó la Perfectionistic Self-Presentation Scale Junior Form (PSPS-Jr) y el Inventario de Ansiedad escolar para Educación Primaria (IAEP). Los análisis estadísticos revelaron una positiva y significativa asociación entre la APP y la Ansiedad Escolar en todos sus factores y el total de sus escalas, con una elevada correlación entre la No-Divulgación de la Imperfección y la Ansiedad ante el Castigo Escolar. Atendiendo a estas consideraciones, este estudio evidencia características específicas que determinan el diseño de un posible tratamiento para disminuir rasgos desadaptativos de la personalidad en población infanto-juvenil.

Palabras clave: autopresentación perfeccionista; PSPS-Jr, ansiedad escolar; Educación Primaria; infancia 


\title{
AUTOPRESENTACIÓN PERFECCIONISTA DURANTE LA INFANCIA Y SU ASOCIACIÓN CON ANSIEDAD ESCOLAR
}

\begin{abstract}
Perfectionist self-presentation during chilhood and its association with school anxiety.

Perfectionist Self-Presentation (PSP), known as the desire to project a perfect image towards others with protective characteristics of its social exhibition and with the reiterative need to divulge excellent, is one of the most influential in psychopathology derived factors, and one of the less studied in the educational context. The aim of this study was to examine the relationship between PSP and School Anxiety in a sample of 860 participants aged between 8 and 12 years $(M=9.86 ; S D=$ 1.26), registered in public and private centers of the province of Alicante. We used the Perfectionistic Self-Presentation Scale Junior Form (PSPS-Jr) and the Inventario de Ansiedad escolar para Educación Primaria (IAEP). Statistical analyzes revealed a positive and significant association between PSP and School Anxiety in all of its factors and the total of its scales, with a high correlation between Non-Disclosure of Imperfection and Anxiety in School Punishment. Taking to these considerations, this study evidences specific characteristics that determine the design of a possible treatment to decrease maladaptive personality traits in the child and youth population.
\end{abstract}

Keywords: perfectionist self-presentation; PSPS-Jr; school anxiety; Primary Education; chilhood

Los individuos perfeccionistas suelen ser vistos como personas que ambicionan una aprobación y reconocimiento social, dirigiendo positivamente sus objetivos hacia una lucha constante orientada al logro. Desgraciadamente, esta percepción elevadamente depurada, no contempla algunos aspectos problemáticos del perfeccionismo (Flett, Hewitt y Sherry, 2016). El perfeccionismo es un rasgo de la personalidad que, a menudo, afecta de modo negativo en la vida de los sujetos que lo padecen, y es caracterizado por un excesivo sacrificio personal orientado a objetivos de elevado rendimiento, acompañando autoevaluaciones críticas exageradas e inquietudes con respecto a las apreciaciones ajenas (Crãciun y Holdevici, 2012). De ahí que se conceptualice como un constructo multidimensional (Hewitt, Flett, Turnbull-Donovan y Mikail, 1991) integrando elementos intrapersonales, interpersonales y autopresentaciones que derivan de componentes sociales (Hewitt, Flett y Ediger, 1995). En este caso concreto, centraremos nuestro estudio en el tercero de los componentes.

La Autopresentación Perfeccionista (APP) es conocida como el deseo de proyectar una imagen perfecta hacia los demás (Chen, Hewitt, Flett, Cassels y Birch, 2012; Hewitt et al., 1995; Hewitt, Flett, Sherry y Caelian, 2006) con características protectoras de su exhibición social y con la necesidad reiterativa de divulgarse excelente (Flett y Hewitt, 2013).

La APP se compone de tres factores: la Auto-Promoción Perfeccionista (AP), la No-Visualización de la Imperfección (NVI) y la No-Divulgación de la Imperfección (NVI) (Hewitt et al., 2003; Hewitt et al., 2011). La AP capta la tendencia a tratar de pregonar continuamente una absoluta brillantez, Io que refleja una preocupación excesiva sobre la presentación perfecta de uno mismo y la carencia de una manifestación realista; la segunda de ellas, la NVI, se basa en encubrir la exteriorización de conductas inexactas, evitando situaciones deficientes y preocupándose excesivamente por cometer algún error en público. Por último, la NDI consiste en la tendencia a no verbalizar la imperfección personal, caracterizándose por la carencia de expresión de cualquier problemática inherente (Hewitt et al., 2003, 2011). Consecuentemente, el conjunto de estos factores, suponen una gran angustia psicológica (Hewitt et al., 2003).

En lo que respecta a población infanto-juvenil, diversos estudios han demostrado que este rasgo de la personalidad se relaciona positivamente con una gran cantidad de problemas de desadaptación y trastornos psicológicos (Flett, Coulter y Hewitt, 2012). Diferentes autores hallaron diferencias entre facetas de la APP entre niños y adultos (Hewitt et al., 2003, 2011; Sherry, Hewitt, Flett, 
Lee-Baggley y Hall, 2007), siendo los estudios de perfeccionismo infanto-juvenil escasos, sobre todo en cuanto se refiere a investigaciones centradas en el ámbito psicopatológico (Vicent, Inglés, Gonzálvez, Sanmartín y García-Fernández, 2016; García-Fernández et al., 2016).

En población infanto-juvenil, la APP ha sido asociada positiva y significativamente con el narcisismo patológico (Borroni et al., 2016), la anorexia nerviosa (Castro et al., 2004), dimensiones del perfeccionismo como el Perfeccionismo Auto-Orientado (PA0), el Perfeccionismo Socialmente Prescrito (PSP) y el Perfeccionismo Orientado a los Demás (POD), así como con depresión, personalidad psicopática, implacabilidad y carencia de emotividad (Hewitt et al., 2011), ansiedad social, actitud disfuncional (Flett et al., 2012), sentimiento angustiado (Flett y Hewitt, 2013), suicidio en la juventud (Flett et al., 2012; Flett y Hewitt, 2013; Hewitt et al., 2011) y Trastornos de Conducta Alimentaria (TCA; Castro et al., 2004; Flett et al., 2012).

En esta misma línea, también en población infanto-juvenil se ha asociado negativamente con variables de ajuste y adaptación como por ejemplo la autoestima (Stoeber y Roche, 2014). Asimismo, estudios realizados por Hewitt et al. (2011) observaron correlaciones negativas y significativas entre NDI y la expresión emocional y emotividad, hallazgos que los autores asocian a la carencia de habilidad social y el sentido de identidad personal de estos sujetos. Además, el nivel de auto-encubrimiento que singulariza a estos individuos puede llegar a no revelar su sufrimiento, incluso cuando padecen un gran pesar psicológico (Cepeda-Benito y Short, 1998; Flett y Hewitt, 2013).

\section{EL PRESENTE ESTUDIO}

El presente trabajo trata de analizar la relación entre el PSPS-Jr y los distintos factores que componen la Ansiedad escolar durante la etapa de Educación Primaria, entendiendo esta como como un conjunto de respuestas cognitivas, motoras y psicofisiológicas emitidas por un sujeto ante condiciones escolares evaluadas como amenazantes, confusas o peligrosas (García-Fernández, Inglés, Martínez-Monteagudo y Redondo, 2008; Martínez-Monteagudo, Inglés, Cano-Vindel y GarcíaFernández, 2012). Así, se espera que las puntuaciones de la APP correlacionen significativa y positivamente con las dimensiones de la ansiedad escolar, en consonancia con investigaciones previas que vinculan la APP con otros tipos de ansiedad, como la ansiedad social (Flett, Hewitt, Blankstein, Solnik y Van Brunschot, 1996; Flett et al., 2012; Hewitt et al., 2003, 2011; Mackinnon, Battista, Sherry y Stewart, 2014; Stoeber y Roche, 2014), y la fobia social (Jain y Sudhir, 2010).

\section{MÉTODO}

\section{Participantes}

La muestra fue seleccionada bajo criterio de accesibilidad. Concretamente, se obtuvieron 937 alumnos de $3^{\circ}, 4^{\circ}, 5^{\circ}$ y $6^{\circ}$ curso de Educación Primaria, con edades comprendidas entre los 8 y 12 años, matriculados en 9 centros públicos y privados de la provincia de Alicante, situados en contextos rurales y con un nivel cultural y socioeconómico de carácter medio. Sin embargo, fueron excluidos 77 participantes por no haber recibido el consentimiento de sus tutores, por omisiones en sus respuestas o por no poseer el nivel mínimo de lectura requerido. De manera que, la muestra definitiva quedó compuesta por 860 participantes $(M=9.86$; $D T=1.26)$, de los cuales 485 pertenecían al sexo femenino y 375 al masculino. En cuanto a la distribución de la muestra en función del curso se obtuvo que un $47.1 \%, 29.1 \%, 12.8 \%$, y $11.0 \%$ de los participantes cursaban $3^{\circ}, 4^{\circ}, 5^{\circ}$ y 60 curso de Educación Primaria, respectivamente. La prueba $\chi^{2}$ de homogeneidad de la distribución de frecuencias reveló la ausencia de diferencias estadísticamente significativas entre los ocho grupos de Sexo x Curso $\left(\chi^{2}=3.62, p=.30\right)$. 


\section{AUTOPRESENTACIÓN PERFECCIONISTA DURANTE LA INFANCIA Y SU ASOCIACIÓN CON ANSIEDAD ESCOLAR}

\section{Medidas}

Perfectionistic Self-Presentation Scale-Junior Form

Perfectionistic Self-Presentation Scale-Junior Form (PSPS-Jr; Hewitt et al., 2011) es una medida de la APP, adaptada a población infantojuvenil. Este instrumento fue constituido mediante la Perfectionistic Self-Presentation Scale (PSPS, Hewitt et al., 2003) creada para medir las tres facetas de APP en adultos: (a) Autopromoción Perfeccionista, (b) No-Visualización de la Imperfección, y c) No-Divulgación de la Imperfección, mediante 27 ítems.

Hewitt et al. (2011) adaptaron la PSPS-Jr a una escala de autoinforme compuesta por 18 ítems con respuesta tipo Likert ( $1=$ Totalmente en desacuerdo; $5=$ Totalmente de acuerdo) y validada por los mismos autores. Dicho instrumento evalúa, de igual manera, los tres factores de la APP. Estos se dividen en: ocho ítems que miden la I. Autopromoción Perfeccionista (AP; e.g., Es importante actuar perfectamente cuando estoy con otras personas) ( $\alpha=.92)$, seis para la II. No-Visualización de la Imperfección (NVl; e.g., Pienso mucho sobre los errores que he cometido delante de otras personas) ( $\alpha=.82$ ) y cuatro para la III. No-Divulgación de la Imperfección (NDI; e.g., Cuando fallo en algo, no permito que otras personas lo sepan) $(\alpha=.72)$ (Hewitt et al., 2011).

\section{Inventario de Ansiedad Escolar para Educación Primaria (IAEP)}

El Inventario de Ansiedad Escolar para Educación Primaria (IAEP) (Gómez-Núñez, 2015) fue construido mediante una adaptación del Inventario de Ansiedad Escolar para Educación Secundaria y Bachillerato (IAES; García-Fernández, Inglés, Martínez-Monteagudo, Marzo y Estévez, 2011), con el objetivo del análisis de la ansiedad escolar durante la etapa de $2^{\circ}$ y $3^{\circ}$ ciclo en Educación Primaria (8 a 11 años de edad). El IAEP consta de 22 ítems, con respuesta tipo Likert de 5 puntos $(0=$ Nada pertinente; 4 = Muy pertinente), referentes a cuatro situaciones escolares que pueden provocar ansiedad, y 15 ítems que presentan las diversas respuestas de ansiedad escolar. Los factores situacionales se componen de: I. Ansiedad ante el Castigo Escolar (e.g., Si no llevo los deberes hechos); II. Ansiedad ante la Victimización (e.g., Si se ríen de mí en el colegio); III. Ansiedad ante la Evaluación Social (e.g., Salir a la pizarra); y IV. Ansiedad ante la Evaluación Escolar (e.g., Cuando me van a dar la nota de un examen). Por otro lado, las escalas de respuesta aluden a manifestaciones cognitivas (e.g., Me siento culpable), conductuales (e.g., No puedo estarme quieto) y psicofisiológicas (e.g., Mi respiración se hace más rápida).

Los niveles de fiabilidad fueron aceptables tanto para el total de la escala $(\alpha=.92)$ como para los cuatro factores situacionales ( $\alpha=$ entre .85 y .89) y las tres escalas de respuesta ( $\alpha=$ entre .80 y .84) (Gómez-Núñez, 2015).

\section{Procedimiento}

Se llevó a cabo una entrevista con el equipo directivo de los centros participantes con la finalidad de exponer el objetivo de la investigación, describir el instrumento de evaluación y solicitar permiso y colaboración. Del mismo modo, fue solicitado el consentimiento informado por escrito a los tutores legales de todos los participantes del estudio, para autorizar a sus hijos a colaborar en la investigación. Una vez concluido el proceso de selección de la muestra de participantes y la obtención del consentimiento informado de los mismos, se dio paso a la administración de las pruebas. Para ello, fue elaborado un cuaderno con las tres escalas de medida, con el objetivo de facilitar la tarea y evitar pérdidas o devoluciones incompletas.

El instrumento se administró de manera voluntaria y colectiva en el aula, contando con la ayuda de los tutores y tutoras de los respectivos grupos. Una vez explicadas las instrucciones pertinentes, se indicó, en primer lugar, que cumplimentasen los datos demográficos del estudiantado, y se acentuó la relevancia de no dejar ítems sin respuesta. 
La administración de las pruebas se llevó a cabo en una sesión de cuarenta y cinco minutos. La investigadora estuvo presente durante el proceso con el objetivo de aclarar posibles dudas y asegurar la correcta aplicación de los datos.

\section{Análisis estadísticos}

Análisis de datos

Las correlaciones entre las puntuaciones del PSPS-Jr y el IAEP se observaron utilizando coeficientes de correlación producto-momento de Pearson. Según Cohen (1988) valores entre .10 y .30 se entienden como un índice de relación de pequeña magnitud, mientras que coeficientes entre .30 y .50 indican una relación de magnitud moderada, y valores superiores a .50 indican una correlación de elevada magnitud. Para los análisis contemplados se utilizaron los programas estadísticos SPSS versión 22.

\section{RESULTADOS}

Respecto a las correlaciones entre los factores y la puntuación total del IAEP con los factores del PSPS-Jr (véase Tabla 1), la correlación más alta se da entre el Factor I (Ansiedad ante el Castigo Escolar) del IAEP y la NDI del PSPS-Jr $(r=.84)$ siendo esta de elevada magnitud, según los criterios de Cohen (1988).

Tabla 1.

Correlaciones entre los distintos factores situacionales del PSPS-Jr con el IAEP (ansiedad escolar).

\begin{tabular}{|c|c|c|c|c|c|}
\hline \multirow{9}{*}{ IAEP } & Factores & FIAPP & FIIAPP & FIIIAPP & TotalAPP \\
\hline & FIIAEP & $.33 * *$ & $.35 * *$ & $.84 * *$ & $.76^{* *}$ \\
\hline & FIIIAEP & $.26^{*}$ & $.22 *$ & $.64 * *$ & $.58 * *$ \\
\hline & FIIIIAEP & $.26^{*}$ & $.44 * *$ & $.63 * *$ & $.57 * *$ \\
\hline & FIVIAEP & $.31 * *$ & $.33 * *$ & $.69 * *$ & $.66^{* *}$ \\
\hline & ACog & $.33 * *$ & $.36 * *$ & $.79 * *$ & $.73 * *$ \\
\hline & ACon & $.32 * *$ & $.32 * *$ & $.77 * *$ & $.71 * *$ \\
\hline & APsicf & $.29 * *$ & $.36^{* *}$ & $.76^{* *}$ & $.70 * *$ \\
\hline & TotIAEP & $.34 * *$ & $.38 * *$ & $.83 * *$ & $.76^{* *}$ \\
\hline \multicolumn{6}{|c|}{$\begin{array}{l}\text { Nota: } * p<.05 ; * * p=.01 \text {; FIIAEP }=\text { Ansiedad ante el Castigo } \\
\text { Escolar; FIIIAEP }=\text { Ansiedad ante la Victimización; FIIIIAEP = } \\
\text { Ansiedad ante la Evaluación Social: FIVIAEP = Ansiedad ante la } \\
\text { Evaluación Escolar; ACog = Ansiedad cognitiva; ACon = Ansiedad } \\
\text { conductual; APsicf = Ansiedad Psicofisiológica; TotIAEP = } \\
\text { Puntuación Total del IAEP; FIAPP = Autopromoción } \\
\text { Perfeccionista; FIIAPP = No-Visualización de la Imperfección; } \\
\text { FIIIAPP = No-Divulgación de la Imperfección; TotalAPP = Total de } \\
\text { la Escala PSPS-Jr. }\end{array}$} \\
\hline
\end{tabular}

Este tercer factor del PSPS-Jr correlacionó de modo más elevado con los diversos factores y el total del IAEP ( $r=$ entre .63 y.84). Por el contrario, la correlación más baja fue presentada entre el Factor II (Ansiedad ante la Victimización) del IAEP y la NVI del PSPS-Jr $(r=.22)$. Sin embargo, de manera general, se obtuvieron puntuaciones menos elevadas entre el IAEP y la AP del PSPS-Jr $(r=$ entre .26 y.33). 


\section{AUTOPRESENTACIÓN PERFECCIONISTA DURANTE LA INFANCIA Y SU ASOCIACIÓN CON ANSIEDAD ESCOLAR}

Todas las correlaciones entre los factores y la puntuación total del IAEP con los factores del PSPS-Jr fueron significativas (véase Tabla 1).

\section{DISCUSIÓN}

En este trabajo se propuso examinar la relación entre la APP y la ansiedad escolar en población infantil. En lo que respecta al PSPS-Jr, tal y como se esperaba, se observan correlaciones estadísticamente significativas y de magnitud elevada con la ansiedad escolar, confirmándose la hipótesis planteada en el estudio. Estos resultados coinciden con investigaciones anteriores realizadas entre APP y ansiedad social, en las que se observa una asociación significativa y en sentido positivo entre ambos constructos (Flett et al., 1996; Flett et al., 2012; Hewitt et al., 2003; 2011; Mackinnon et al., 2014; Stoeber y Roche, 2014).

Igualmente, cabe destacar las correlaciones de moderada y elevada magnitud, observadas entre la NDI y el total del PSPS-Jr. La estrecha relación que se observa entre la dimensión de NDI y la ansiedad escolar se encuentra en la misma línea que otros trabajos elaborados con adultos que demuestran que dicha faceta de la APP está fuertemente vinculada con una excesiva ansiedad social (Hewitt et al., 2003) y con índices elevados de angustia, ya que la NDI representa una desadaptación de su propia gestión de imperfección (Hewitt et al., 2011). Por tanto, la relación entre la NDI y la ansiedad escolar podría explicarse, por un lado, debido al temor que manifiestan estos sujetos hacia la posible observación pública de cualquier imperfección, cuestión que causa una gran correlación significativa y positiva con la ansiedad social y el perfeccionismo (Flett, Greene y Hewitt, 2004; Inglés, García-Fernández, Vicent, Gonzálvez y Sanmartín, 2016). Debido al temor hacia la contemplación de imperfecciones, que expresan los autores, desde la correlación entre ansiedad social y el perfeccionismo, se produciría el mismo sentimiento hacia cualquier divulgación de estas, observando nexos de unión con el rasgo interpersonal del perfeccionismo (APP), y con mayor detalle en el tercer factor de la APP (NDI). Por otro lado, dicha relación también se justifica por la generación de ansiedad social al considerar que una impresión imperfecta puede tener consecuencias negativas, en consonancia con investigaciones realizadas por Mackinnon et al. (2004). Sin embargo, todas estas aportaciones, teniendo en cuenta los estudios previos expresados, surgen como hipótesis complementarias, por lo que se sugiere ampliar la investigación dentro de este campo de estudio.

Cabe mencionar que estos resultados no son generalizables a otros grupos que no sean sujetos entre 8 y 12 años. Por tanto, convendría que futuros trabajos analizasen la asociación entre el PSPSJr y la Ansiedad escolar en población española adolescente. Igualmente, el diseño del estudio nos impide establecer relaciones causales entre las variables estudiadas. Aspecto que podrían solventar futuros trabajos empleando el método de ecuaciones estructurales.

\section{CONCLUSIONES}

Atendiendo a estas consideraciones, este estudio evidencia relevantes implicaciones para la comprensión de la APP durante la infancia, examinando por primera vez la relación entre la APP y los distintos factores de la Ansiedad escolar. Los resultados sugieren un patrón de asociación significativa y positiva en todas sus dimensiones. Contemplando la relevancia de este rasgo de la personalidad y la comorbilidad de psicopatologías observadas en el estudio tales como el sentimiento angustiado (Besser, Flett y Hewitt, 2010; Flett y Hewitt, 2013; Sherry et al., 2007), ansiedad (Flett et al., 1996; Hewitt et al., 2003, 2011; Mackinnon et al., 2014; Stoeber y Roche, 2014), fobia social y bienestar subjetivo (Casale, Fioravanti, Flett y Hewitt, 2015; Jain y Sudhir, 2010; Mackinnon y Sherry, 2012), baja autoestima y depresión (Beser et al., 2010; Crãciun y Holdevici, 2012; Stoeber y Roche, 2014), TCA (Geller, Cockell, Hewitt, Goldner y Flett, 2000; Hewitt et al., 1995) y tendencias suicidas (Chang, 2002; Flett y Hewitt, 2013; Hassan, Flett, Ganguli y Hewitt, 2014; Roxborough et al., 2012), 
y dada la escasez de estudios elaborados hasta el momento sobre APP infantil, se considera imprescindible el aumento de investigaciones en esta línea, en los años posteriores.

\section{REFERENCIAS BIBLIOGRÁFICAS}

Besser, A., Flett, G. L., y Hewitt, P. L. (2010). Perfectionistic self-presentation and trait perfectionism in social problem-solving ability and depressive symptoms. Journal of Applied Social Psychology, 40 (8), 2121-2154. doi:10.1111/j.1559-1816.2010.00653.x

Borroni, S., Bortolla, R., Lombardi, L.M., Somma, A., Maffei,C., y Fossati, A. (2016). The Italian version of Perfectionistic Self-Presentation Scale: Psychometric proprieties and its associations with pathological narcissism and adult attachment in an adult non clinical simple. Personal Men Health, 10 (2), 130- 141.

Casale, S., Fioravanti, G., Flett, G.L., y Hewitt, P.L. (2015). Self-presentation styles and Problematic use of Internet communicative services: The role of the concerns over behavioral displays of imperfection. Personality and Individual Differences, 76, 187-192.

Castro, J., Gila, A., Gual, P., Lahortiga, F., Saura, B. A., y Toro, J. (2004). Perfectionism dimensions in children and adolescents with anorexia nervosa. Journal of Adolescent Health, 35, 392-398.

Crãciun, B., y Holdevici, I. (2012). Cognitive-Behavioural Intervention in Diminishing Perfectionistic Self-Presentation and Depression. Procedia-Social and Behavioral Sciences, 78, 476-480.

Cepeda-Benito, A., y Short, P. (1998). Self-concealment, avoidance of psychological services, and perceived likelihood of seeking professional help. Journal of Counseling Psychology, 45, 58-64.

Chen, C., Hewitt, P.L., Flett, G., Cassels, T.G., y Birch, S. (2012). Insecure attachment, perfectionistic selfpresentation, and social disconnection in adolescents. Personality and Individual Differences, 52, 936-941.

Chang, E. C. (2002). Examining the link between perfectionism and psychological maladjustment: Social problem-solving as a buffer. Cognitive Therapy and Research, 26, 581-595.

Hewitt, P. L., Flett, G. L., Turnbull-Donovan, W., y Mikail, S. F. (1991). The Multidimensional Perfectionism Scale: Reliability, Validity, and Psychometric Properties in Psychiatric Samples. Psychological Assessment: A Journal of Consulting and Clinical Psychology, 3 (3), 464-468.

Hewitt, P. L., Flett, G. L., y Ediger, E. (1995). Perfectionism traits and perfectionistic self-presentation in eating disorder attitudes, characteristics, and symptoms. International Journal of Eating Disorders, 18, 317-326.

Hewitt, P., Flett, G.L., Sherry, S. B., Habke, M., Parkin, M., Lam, R.W.,...Stein, M. B. (2003). The Interpersonal Expression of Perfection: Perfectionistic Self- Presentation and Psychological Distress. Journal of Personality and Social Psychology, 84 (6), 1303-1325.

Hewitt, P. L., Flett, G. L., Sherry, S. B., y Caelian, C. (2006). Trait perfectionism and suicide behavior. En T. E. Ellis (Ed.), Cognition and suicide: Theory, research, and practice (pp. 215-235). Washington, DC: American Psychological Association.

Hewitt, P. L., Blasberg, J. S., Flett, G. L., Besser, A., Sherry, S. B., Caelian, C., y Birch, S. (2011). Perfectionistic self-presentation in children and adolescents: Development and validation of the Perfectionistic Self-Presentation Scale-Junior Form. Psychological Assessment, 23, 125-142.

Inglés, C. J., García-Fernández, J. M., Vicent, M., Gonzálvez, C. y Sanmartín, R. (2016). Profiles of Perfectionism and School Anxiety: A Review of the $2 \times 2$ Model of Dispositional Perfectionism in Child Population. Frontiers in Psychology, 7 (1403). doi: 10.3389/fpsyg.2016.01403

Flett, G. L., Greene, A., y Hewitt, P. L. (2004). Dimensions of perfectionism and anxiety sensitivity. Journal of Rational-Emotive and Cognitive-Behavior Therapy, 22, 37-55.

Flett, G. L., Hewitt, P. L., Blankstein, K. R., Solnik, M., y Van Brunschot, M. (1996). Perfectionism, 


\section{AUTOPRESENTACIÓN PERFECCIONISTA DURANTE LA INFANCIA Y SU ASOCIACIÓN CON ANSIEDAD ESCOLAR}

social problem-solving ability, and psychological distress. Journal of Rational-Emotive and Cognitive-Behavior Therapy, 14, 245-275.

Flett, G. L., Coulter, L.M., y Hewitt, P. L. (2012). The Perfectionistic Self-Presentation Scale-Junior Form: psychometric properties and association with social anxiety in early adolescents. Canadian Journal of School Psychology, 27 (2) 136-149. doi:10.1177/0829573511431406

Flett, G. L., y Hewitt, P. L. (2013). Disguised Distress in Children and Adolescents "Flying Under The Radar": Why Psychological Problems Are Underestimated and How Schools Must Respond. Canadian Journal of School Psychology, 28 (1) 12-27.

Flett, G., Hewitt, P. L., y Sherry, S. (2016). Deep, dark, and dysfunctional: The destructiveness of interpersonal perfectionism. En V. Zeigler-Hill y D. K. Marcus (Ed.), The dark side of personality: Science and practice in social, personality, and clinical psychology (pp.211-229). Washington: American Psychological Association.

García-Fernández, J.M., Inglés, C.J., Martínez-Monteagudo, M.C., y Redondo, J. (2008). Evaluación y tratamiento de la ansiedad escolar en la infancia y la adolescencia. Behavioral Psychology/Psicología Conductual, 16, 413-437.

García-Fernández, J. M., Inglés, C. J., Martínez-Monteagudo, M.C., Marzo, J.C., y Estévez, E. (2011). Inventario de Ansiedad Escolar: validación en una muestra de estudiantes de Educación Secundaria. Psicothema, 23 (2), 301-307.

García-Fernández, J. M., Inglés, C. J., Vicent, M., Gonzálvez, C., Gómez-Núñez, M. I. y Poveda-Serra, P. (2016). Perfeccionismo durante la infancia y la adolescencia. Análisis bibliométrico y temático (2004-2014). [Perfectionism during childhood and adolescence. Bibliometric and thematic analysis (2004-2014)]. Revista Iberoamericana de Psicología y Salud, 7, 79-88. doi: 10.1016/j.rips.2016.02.001

Geller, J., Cockell, S. J., Hewitt, P. L., Goldner, E. M., y Flett, G. L. (2000). Inhibited expression of negative emotions and interpersonal orientation in anorexia nervosa. International Journal of Eating Disorders, 28, 8-19.

Gómez-Núñez, M.I (2015). Elaboración y validación de un cuestionario para evaluar la Ansiedad Escolar en la infancia tardía (Tesis doctoral inédita). Psicología evolutiva y didáctica, Universidad de Alicante.

Hassan, S., Flett, G.L., Ganguli, R., y Hewitt, P. (2014). Perfectionistic Self-Presentation and Suicide in a Young Woman with Major Depression and Psychotic Features. Case Reports in Psychiatry, 1-6.

Jain, M., y Sudhir, P. M. (2010). Dimensions of perfectionism and perfectionistic self-presentation in social phobia. Asian Journal of Psychiatry, 3, 216-221.

Mackinnon, S. P., y Sherry, S. B. (2012). Perfectionistic self-presentation mediates the relationship between perfectionistic concerns and subjective well-being: A three-wave longitudinal study. Personality and Individual Differences, 53, 22-28.

Mackinnon, S. P., Battista, S. R., Sherry, S. B., y Stewart, S. H. (2014). Perfectionistic self-presentation predicts social anxiety using daily diary methods. Personality and Individual Differences, 56, 143-148.

Martínez-Monteagudo, M.C., Inglés, C. J., Cano-Vindel, A., y García-Fernández, J.M. (2012). Estado actual de la investigación sobre la teoría tridimensional de la ansiedad de Lang. Ansiedad y Estrés, 18 (2-3), 201-219.

Roxborough, H. M., Hewitt, P. L, Kaldas, J., Flett, G.L., Caelian, C.M., Sherry, S., y Sherry, D. (2012). Perfectionistic self-presentation, socially prescribed perfectionism, and suicide in youth: a test of the perfectionism social disconnection model. Suicide Life Threat Behavior, 42 (2), 217-233. 
Sherry, S.B., Hewitt, P. L., Flett, G.L., Lee-Baggley, D. L., y Hall, P. A. (2007). Trait perfectionism and perfectionistic self-presentation in personality pathology. Personality and Individual Differences, 42, 477-490.

Stoeber, J., y Roche, D. L. (2014). Affect intensity contributes to perfectionistic self-presentation in adolescents beyond perfectionism. Journal of Rational-Emotive and Cognitive- Behavior Therapy, 32, 164-180. doi: 10.1007/s10942-013-0176- x

Vicent, M., Inglés, C. J., Gonzálvez, C., Sanmartín, R., García-Fernández, J. M. (2016). Perfeccionismo socialmente prescrito y los cinco grandes rasgos de la personalidad en niños españoles. European Journal of Investigation in Health, Psychology and Education, 6 (2), 107118. 
